\title{
Venous Thromboembolism and D-dimer In Patients with COVID-19
}

\author{
Joshua T. Hoffer ${ }^{1}$, Lauren K. Stewart ${ }^{2}$, Kate L. Pettit ${ }^{2}$, Jeffrey A. Kline ${ }^{2}$ \\ ${ }^{1}$ Indiana University School of Medicine ${ }^{2}$ Indiana University School of Medicine, Department of \\ Emergency Medicine
}

Background and Hypothesis: The potential association between venous thromboembolism (VTE) and COVID-19 is an area of growing research, and methods of effective prophylaxis, detection, and treatment continue to be sought. D-dimer assays have been previously established as a highly sensitive - albeit nonspecific - test to assess patient risk of VTE, but the full clinical utility of this test in COVID-19 patients is currently not well understood. We hypothesized that, in patients presenting to the emergency department (ED) and determined to be positive for COVID-19, an elevated D-dimer value is associated with an increased 30day incidence of VTE.

Project Methods: Deidentified patient encounter data was collected and analyzed from a multicenter registry of ED patients tested for SARS-CoV-2. We measured the frequency of a positive SARS-CoV-2 test and compared the incidence of VTE between SARS-CoV-2 positive and negative patients. We also compared average D-dimer values in SARS-CoV-2 positive patients with and without VTE.

Results: Of 6,445 patient encounters queried, 2,051 tested positive for SARS-CoV-2 (32\%). SARS-CoV-2 positive patients had a significantly higher incidence of VTE in the 30-day followup period compared to SARS-CoV-2 negative patients (3.2 vs. $1.6 \%, p=0.0002$ ). D-dimer values were available for 537 of these SARS-CoV-2 patients, with an average D-dimer of $1813.83 \mathrm{ng} / \mathrm{mL}$. The average D-dimer in SARS-CoV-2 positive patients who did develop VTE tended to be higher than those who did not develop VTE $(2969 \mathrm{ng} / \mathrm{mL}$ vs. $1822 \mathrm{ng} / \mathrm{mL})$, although this difference was not statistically significant $(p=0.34)$. The fitted areas for binomial receiver operating characteristic curves of D-dimer for detection of VTE in patients with and without SARS-CoV-2 were 0.628 and 0.829 , respectively.

Conclusion and Potential Impact: We found a positive SARS-CoV-2 test to be associated with a higher incidence of VTE. However, an elevated D-dimer continues to be nonspecific for VTE in SARS-CoV-2 positive patients, and performs more poorly in SARS-CoV-2 positive patients than in negative patients. Additional clinical criteria should be identified to further guide the use of diagnostic imaging modalities and prophylactic anticoagulation in COVID-19 positive patients with suspicion of VTE. 